\title{
BMI open Empirical evidence that disease prevalence may affect the performance of diagnostic tests with an implicit threshold: a cross-sectional study
}

To cite: Willis BH. Empirical evidence that disease prevalence may affect the performance of diagnostic tests with an implicit threshold: a cross-sectional study. BMJ Open 2012;2: e000746. doi:10.1136/ bmjopen-2011-000746

- Prepublication history and additional appendix for this paper are available online. To view these files please visit the journal online (http:// bmjopen.bmj.com).

Received 9 December 2011 Accepted 20 December 2011

This final article is available for use under the terms of the Creative Commons Attribution Non-Commercial 2.0 Licence; see http://bmjopen.bmj.com
Department of Biostatistics, University of Manchester, Manchester, UK

Correspondence to Dr Brian H Willis; b.h.willis@doctors.org.uk

\section{ABSTRACT}

Objective: To investigate the effects that prevalence has on the diagnostic performance of junior doctors in interpreting $x$-rays.

Design: Two-armed cross-sectional design using systematic sampling.

Setting: Emergency department in the UK.

Participants: From a sample of 2593 patients (1434 men and 1159 women) taken from an unselected attending cohort between January and April 2002, 967 $x$-rays were analysed. The sex distribution was 558 men and 409 women, and the mean age of those receiving an $\mathrm{x}$-ray was 34.6 .

Interventions: The interpretation of $x$-rays by junior doctors after their triage into high- and low-prevalence populations by radiographers.

Main outcome measures: Sensitivity, specificity, likelihood ratios, diagnostic odds ratios and receiver operator characteristic curve.

Results: There were statistically significant differences in the performance characteristics of junior doctors when interpreting high-probability and low-probability $x$-rays. For the high- and low-probability populations, respectively, the sensitivities were $95.8 \%(95 \% \mathrm{Cl}$ $91.1 \%$ to $98.1 \%)$ and $78.3 \%(95 \% \mathrm{Cl} 65.7 \%$ to $87.2 \%)$ and the specificities were $56.0 \%(95 \% \mathrm{Cl}$ $41.9 \%$ to $69.2 \%)$ and $92.3 \%(95 \% \mathrm{Cl} 90.0 \%$ to $94.2 \%)$. Hierarchical logistic regression showed that the sensitivity did depend on the type of $x$-ray being interpreted but the diagnostic odds ratios did not vary significantly with prevalence, suggesting that doctors were changing their implicit threshold between the two populations along a common receiver operator characteristic curve.

Conclusions: This study provides evidence on how the prevalence may affect the performance of diagnostic tests with an implicit threshold and potentially includes the clinical history and examination. This has implications both for clinicians applying research findings to their practice and the design of future studies.

\section{INTRODUCTION}

It is convenient in the assessment of diagnostic tests to divide the study population into two disjoint subpopulations consisting of

\section{ARTICLE SUMMARY}

Article focus

- The sensitivity, specificity and likelihood ratios of a diagnostic test are often assumed to remain constant even when the prevalence (pre-test probability) of disease changes.

- There is a lack of research in the literature on the effects that the prevalence has on the performance of diagnostic tests particularly those tests with implicit thresholds such as when doctors interpret $x$-rays.

- This study investigates the effects that prevalence has on the diagnostic performance of junior doctors in interpreting $x$-rays.

Key messages

- This study provides empirical evidence that the sensitivity, specificity and likelihood ratios may change with prevalence in diagnostic tests that require subjective interpretation, as in the case of junior doctors examining $x$-rays.

- The most plausible explanation for the effect seems to be doctors modifying their threshold for an abnormal diagnosis based on the results of previous tests.

- These results suggest that likelihood ratios and other test accuracy statistics derived from clinical studies have the potential to be misleading when applying them in practice.

Strengths and limitations of this study

- The study models a large data set collected from a real-life clinical setting and is representative of everyday clinical practice.

- The findings are likely to extend beyond the clinical tests analysed here.

- There is a potential for review bias owing to a lack of blinding between the test and reference standard.

those with disease and those without Leading from this observation, many authors have asserted that performance characteristics, such as the sensitivity and specificity, which are derived from one or other of these 
populations but not both, are independent of the prevalence of disease. ${ }^{1-4}$

This assertion has been questioned by some authors, ${ }^{5-8}$ and circumstances in which a change in prevalence may affect the sensitivity and specificity have been described. ${ }^{5}{ }^{6}$ A Bayesian approach to diagnostic medicine relies on the reported values of the sensitivity, specificity and, hence, likelihood ratios being reproducible in practice. For the evidence-based clinician hoping to apply likelihood ratios reported in published studies to their practice, the potential for them to vary with the prior probability could have a profound effect on the reliability of applying diagnostic test research.

Despite its potential importance, currently there are few studies ${ }^{6-13}$ which have considered the effects of prevalence on a test's performance. Ideally, this would be demonstrated by a study design, which has at least two arms, where prior testing has modified the pre-test probabilities so that they are different for each arm, before the test under investigation is applied. There are instances where this has been done for diagnostic tests, which have an explicit (fixed) threshold for a positive result. ${ }^{14-16}$

By contrast, in tests which have an implicit threshold, such as examining an MRI scan, the operator sets the level of the threshold, usually based on prior training and experience, but potentially in response to prior test results. This latter point seems to have received little attention in the literature. While there are examples of studies which have evaluated the performance of tests combined sequentially, ${ }^{17-20}$ due to limitations in design, ${ }^{17-20}$ the effect that each of the different outcomes of a test may have on the performance of a subsequent test has rarely been estimated. ${ }^{21} 22$

To help address this, the example used here investigates the effect the pre-test probability has on the performance of junior doctors in interpreting plain $\mathrm{x}$ rays in an emergency department (ED) setting, before considering the implications for similar diagnostic tests. This study was part of a larger investigation, which has been published elsewhere. ${ }^{23}$ Although the data were collected in 2002, the lack of research in this area and the continuing relevance of the findings underline the importance of research in this field. Note that pre-test probability and prevalence are used interchangeably.

\section{METHOD}

Between January and April 2002, systematic sampling was used to collect data on an unselected attending cohort of patients at the ED of the Horton Hospital in the UK.

All patients seeing an ED junior doctor underwent a clinical examination to determine whether treatment or further investigation was necessary. As part of their evaluation, some patients were required to have an x-ray, where the type of x-ray received depended on the results of the clinical examination.

Before the junior doctors viewed any x-rays, they were first interpreted then triaged, on the basis of their findings, by one of the departmental radiographers (radiologic technologists). Thus, those x-rays considered abnormal or 'high-probability' x-rays were marked with a red dot by the radiographer otherwise they were left unmarked. All the radiographers had received in-house training in interpreting x-rays.

Each x-ray was then interpreted by one of the ED junior doctors (each with similar training of at least 1year experience post-qualification). All x-rays were then verified by a radiologist and this was the reference standard.

The data collected included the date, patient's age, x-ray type (eg, scaphoid), radiographer's triage result, junior doctor's diagnosis and reference diagnosis. The $\mathrm{x}$-rays were classified by the part of the body irradiated (x-ray type), such as chest x-rays.

Features considered abnormal on an x-ray depended on the x-ray type and included fractures (skeletal x-rays), cardiomegaly (chest x-rays) and dilated bowel (abdominal x-rays), thus covering a range of target disorders and are detailed elsewhere. ${ }^{23}$ In the high-probability (red dot) x-rays, the prevalence of abnormal findings was $77 \%$ compared with $13 \%$ in the 'low-probability' x-rays. Although the junior doctors were aware that a red dot indicated a higher probability of an abnormality, they were not aware of how high this probability was.

\section{Statistical analysis}

Two by two tables were derived for each of the high- and low-prevalence subpopulations. The sensitivity, specificity, likelihood ratios and diagnostic odds ratios (DOR) were used for comparison and a receiver operator characteristic (ROC) curve was constructed. ${ }^{124-27}$

While performance statistics, such as the sensitivity and specificity, could be calculated from pooling the data across all the junior doctors, this does not take into account variation in the performances between junior doctors. Furthermore, it does not allow for the effects of the x-ray type on the performances of individual junior doctors.

Hence, a hierarchical logistic regression model ${ }^{27} 28$ was constructed to study the effects of different covariates on the dependent variables, logit sensitivity and logit specificity. Junior doctors were included in the model as a random effect, and covariates on prevalence, $\mathrm{x}$-ray types and broader groupings of $\mathrm{x}$-ray types were also included.

As any effects of prevalence on performance may be explained by differences in performance across different $\mathrm{x}$-ray types, the interaction between the prevalence and $\mathrm{x}$-ray type was evaluated. Cross-level interactions between explanatory variables were also investigated by allowing the slope to vary across individual doctors. Models were compared using the log likelihood ratio test statistic (LRT), which has an asymptotic $\chi^{2}$ distribution with degrees of freedom (df). ${ }^{27}{ }^{28}$ All analyses were completed using the statistical software R, and statistical significance was set at $\mathrm{p}<0.05$. A full description of the model may be found in the online appendix. 
Table 1 Contingency tables showing the summary totals in each of the cells after pooling all the junior doctors

\begin{tabular}{|c|c|c|c|c|c|c|}
\hline \multicolumn{7}{|c|}{ Pooled data for the junior doctors } \\
\hline & \multicolumn{3}{|c|}{ High-prevalence population (77\%) } & \multicolumn{3}{|c|}{ Low-prevalence population (13\%) } \\
\hline & \multicolumn{3}{|c|}{ Reference standard } & \multicolumn{3}{|c|}{ Reference standard } \\
\hline & Positive & Negative & & Positive & Negative & \\
\hline \multicolumn{7}{|c|}{ Doctor's diagnosis } \\
\hline Positive & 159 & 22 & 181 & 72 & 50 & 122 \\
\hline Negative & 10 & 28 & 38 & 24 & 602 & 626 \\
\hline Totals & 169 & 50 & 219 & 96 & 652 & 748 \\
\hline
\end{tabular}

Note $\mathrm{x}$-rays in the high/low-prevalence population were those interpreted by the radiographer as having a high/low probability of an abnormal feature. The true prevalence is determined by the reference standard.

The type of $\mathrm{x}$-ray a patient receives is, in part, indicative of their morbidity. Thus, the distributions of x-ray types were inspected to give some indication on whether the mix of patients (or patient-mix) varied between the high- and low-prevalence populations. If some x-ray types are more difficult to interpret than others (such as abdominal $\mathrm{x}$-rays compared with tibial $\mathrm{x}$-rays), then differences in the relative proportions of these may explain differences in the performance characteristics.

\section{RESULTS}

There were 1053 x-rays interpreted by 26 ED junior doctors following triage by a radiographer. Eighty-six were excluded due to incomplete information on the radiographers' triage result (28), junior doctors' diagnosis (10) and reference diagnosis (48). The remaining 967 x-rays are analysed in table 1.

The striking feature of these results is the change in sensitivity, specificity and positive likelihood ratio between the low- and high-prevalence populations (table 2). The differences are statistically significant and provide evidence against the null hypothesis that the performance characteristics of junior doctors at interpreting x-rays do not vary with prevalence.

In contrast, the DOR for each of the high- and lowprevalence populations were not statistically significantly different, being very close to each other at 37.3 (95\% CI 3.6 to 101.3 ) and 36.1 (95\% CI 21.0 to 62.3 ), respectively. This is consistent with the null hypothesis that the DOR is constant, which has a bearing on the shape of the ROC curve. A common DOR generates a symmetrical ROC curve, ${ }^{24-26}$ and observing how closely the points are to the curve, this informs a possible cause to the variation in the sensitivity and the specificity, namely a change in the implicit threshold for test positives as applied by the junior doctors (figure 1).

The x-ray distributions for each of the normal and abnormal populations are shown in figures 2 and 3. On inspection, the distributions are broadly similar for the high- and low-prevalence populations in each case, with only chest x-rays being an outlier in figure 3. This would suggest that any differences in performance between the high- and low-prevalence populations are unlikely to be due to differences in the relative proportions of x-ray type.

Table 2 Summary performance estimates given for the independent significant covariate, prevalence. Also given are the estimates of sensitivity for each level of the covariate $\mathrm{x}$-ray group, which was significant for the dependent variable logit (sensitivity)

Model estimates of performance characteristics in significant covariates High prevalence Low prevalence

Sensitivity (\%)

Soft tissue $x$-rays

Appendicular x-rays

Axial skeletal $x$-rays

Summary

Specificity (\%)

Summary

Positive likelihood ratio Summary

Negative likelihood ratio Summary

Diagnostic Odds ratio Summary

$$
\begin{aligned}
& 93.7(79.5 \text { to } 98.3) \\
& 97.3(93.3 \text { to } 99.0) \\
& 58.6(17.3 \text { to } 90.5) \\
& 95.8(91.1 \text { to } 98.1) \\
& 56.0(41.9 \text { to } 69.2) \\
& 2.2 \text { (1.6 to } 3.0) \\
& 0.07(0.03 \text { to } 0.17) \\
& 37.3 \text { (3.6 to } 101.3)
\end{aligned}
$$

68.3 (44.3 to 85.3$)$ 84.0 (70.3 to 92.2$)$ 17.0 (2.4 to 63.1$)$ 78.3 (65.7 to 87.2$)$

$10.2(7.6$ to 13.8$)$

$0.23(0.14$ to 0.38$)$

36.1 (21.0 to 62.3 )

All estimates are derived from the hierarchical regression model and take into account variation in performance between individual doctors and different $x$-ray groups. The covariate $x$-ray group has three levels: soft tissue (chest and abdominal x-rays), appendicular (limbs, hands and feet) and axial (skull, spine and sacrum). Interaction terms were not significant. 95\% Cls are shown in brackets. 


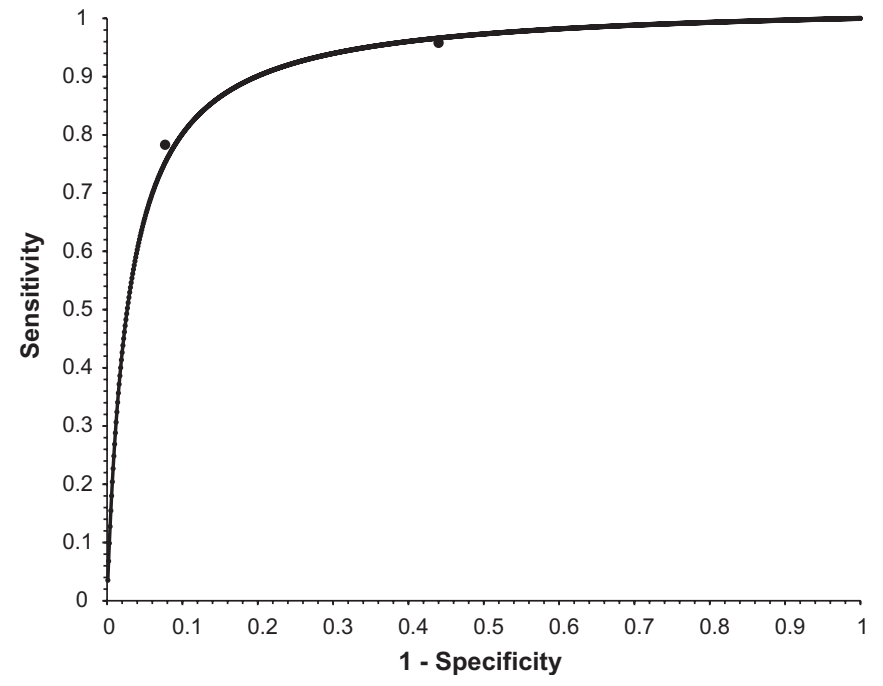

Figure 1 Symmetrical receiver operator characteristic curve (weighted mean diagnostic odds ratios (DOR)) for the average junior doctor. Weighted mean DOR (36.4) was derived from weighting model estimates of DORs for high-prevalence population (37.3) and low-prevalence population (36.1). Point estimates of sensitivity and $1-$ specificity for both populations are also given.

The effects that the change in x-ray distributions may have on performance between the two subpopulations was modelled using hierarchical logistic regression. Unsurprisingly, prevalence was a significant covariate for each of the dependent variables logit sensitivity (LRT=20.6, $\mathrm{df}=1, \quad \mathrm{p} \sim 10^{-5}$ ) and logit specificity (LRT=42.8, $\mathrm{df}=1, \mathrm{p} \sim 10^{-11}$ ).

In contrast, $\mathrm{x}$-ray type was not a significant covariate for either logit sensitivity $(\mathrm{LRT}=34.4, \mathrm{df}=24, \mathrm{p}=0.078)$ or logit specificity ( $\mathrm{LRT}=23.3, \mathrm{df}=33, \mathrm{p}=0.89)$. Owing to the number of levels to the factor x-ray type (34), this could be due to insufficient data. Therefore, x-ray types were grouped into three broad mutually exclusive groups: skeletal $\mathrm{x}$-rays that were subdivided anatomically into appendicular (limbs, hands and feet) and axial (skull, facial and spine) ${ }^{29}$ and soft tissue $\mathrm{x}$-rays (chest and abdomen). The x-ray group was a significant independent covariate for logit sensitivity $(\mathrm{LRT}=10.88, \mathrm{df}=2$, $\mathrm{p}=0.0043$ ) but not for logit specificity (LRT $=2.74, \mathrm{df}=2$, $\mathrm{p}=0.26$ ) (table 2). However, interaction terms between prevalence and x-ray group and across levels between $\mathrm{x}$-ray groups and junior doctors were not significant for either dependent variable.

As chest x-rays were a potential outlier (figure 3), a sensitivity analysis was performed to investigate the effects of this category on the statistical significance of covariates, by including and excluding this category from the model. No significant effects were found.

\section{DISCUSSION}

This study demonstrated statistically significant differences in the sensitivities, specificities and positive likelihood ratios between the high- and low-prevalence populations (table 2), providing evidence that the diagnostic performance of junior doctors in interpreting $\mathrm{x}$ rays does vary with prevalence. There was evidence that the sensitivity depended on the x-rays being interpreted, and although such dependence could not be demonstrated for individual x-ray types (due to sample size), it was demonstrated for broader categories of x-rays. Since the x-ray type is an indicator of the type of target disorder and therefore patient, this implies that the diagnostic performance does depend to some degree on both the type of x-ray being interpreted and the target disorder being sought.

However, this was an independent effect: analysis of the interaction between prevalence and x-ray group was not significant. The effect of the junior doctors' performance varying with prevalence occurred irrespective of the type of x-ray being interpreted or target disorder being sought. Although performance was evaluated over different types of x-rays and multiple target disorders, these findings suggest the potential of observing such
Figure 2 Distribution of x-rays with a normal diagnosis in the two populations: high prevalence (red) and low prevalence (blue). Shown are the percentage of normal $\mathrm{x}$-rays in each population (high or low prevalence), which are of a particular type. For example, $10 \%$ of $x$-rays diagnosed normal in the high-prevalence (red) population were of elbows. Differences in the distributions between the high- and lowprevalence populations could potentially account for differences in the specificity between the respective populations. Note that the normal diagnosis refers to the reference standard diagnosis.

$\mathrm{T} \& \mathrm{~L}$, thoracic and lumbar.

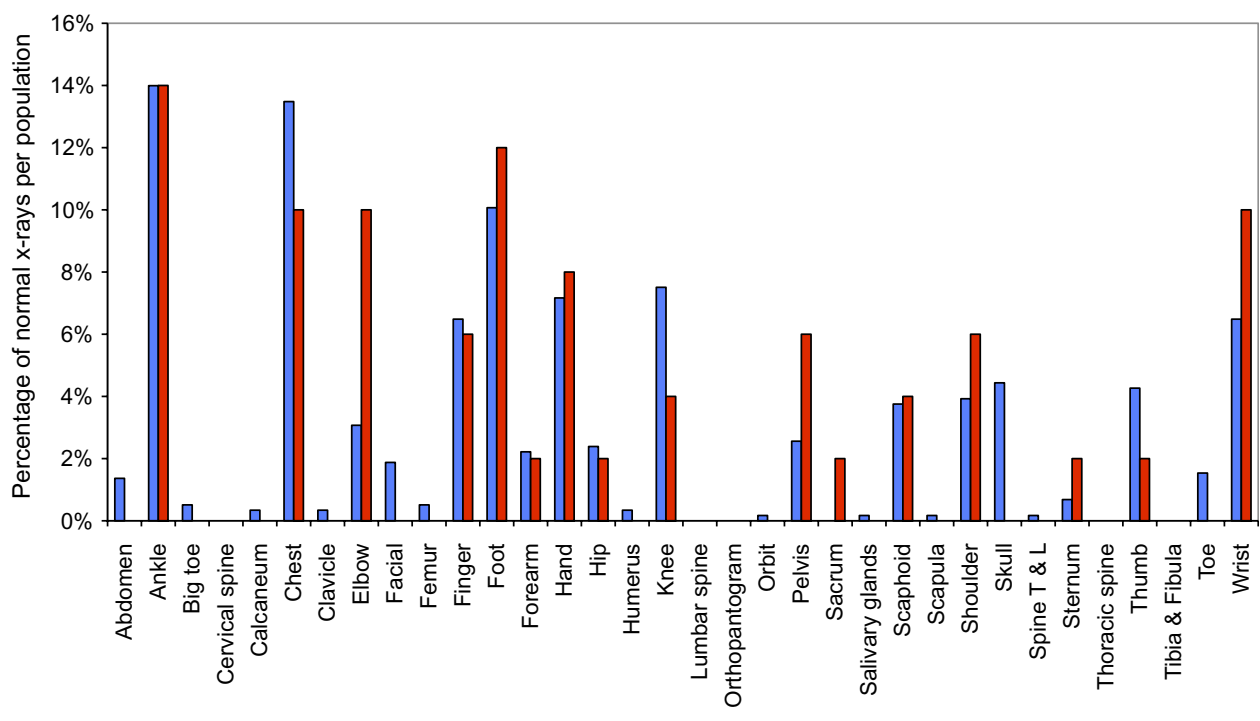


Figure 3 Distribution of $x$-rays with an abnormal diagnosis in the two populations: high prevalence (red) and low prevalence (blue). Shown are the percentage of abnormal x-rays in each population (high or low prevalence), which are of a particular type. For example, $10.5 \%$ of $x$-rays diagnosed abnormal in the high-prevalence (red) population were of ankles. Differences in the distributions between the high- and lowprevalence populations could potentially account for differences in the sensitivity between the respective populations. Note that the abnormal diagnosis refers to the reference standard diagnosis. $T$ \& $L$, thoracic and lumbar.

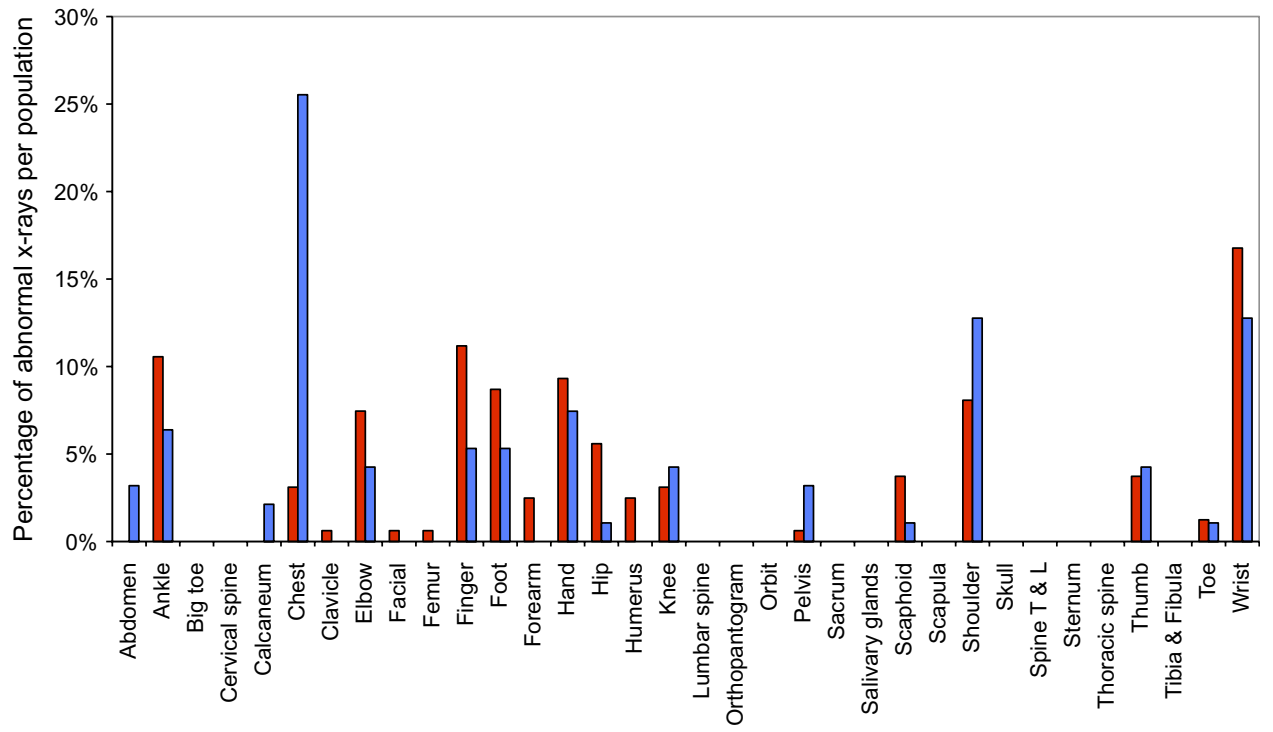

prevalence effects when only a single target condition is of interest.

Given there was insufficient evidence to reject the hypothesis of a common DOR and considering the closeness of the (sensitivity, 1 - specificity) pairs to the ROC curve (figure 1), the doctors' performance seems to change along a single symmetrical ROC curve. This is consistent with the junior doctors changing their implicit threshold for an abnormal diagnosis on the basis of the radiographer's triage result. This does seem plausible when it is noted that the doctors both had knowledge of the previous test's (radiographer's triage) results and could change their subjective threshold for a positive test result on the basis of this information. It is possible that this latter point was amplified by the relative lack of experience in the participating doctors, with more experienced clinicians being expected to exhibit such threshold effects to a lesser degree. Clearly, this study does not answer this latter point.

Other explanations are still possible: the ROC curve may not be unique or symmetrical ${ }^{24-26}$; differences in the patient spectrum between the two populations may affect the different performance characteristics observed. ${ }^{30}{ }^{31}$ For instance, the initial triaging by the radiographers into high- and low-probability x-rays is dependent on their ability to spot abnormal features. Severe cases, where the abnormal features are more striking, are more easily identified and more likely to be allocated to the high-prevalence (probability) population. Thus, the differences in performance between the high and low populations could be a reflection of differences in severity.

This cannot be discounted and almost certainly explains part of the effect of the prevalence on performance. However, the circumstantial evidence in favour of junior doctors changing their implicit threshold seems more extensive, suggesting that this is likely to be the most important factor.
The question that is raised by this example is whether the effects observed may be generalised to other diagnostic tests? An example where these may occur is in the dynamic process of taking a clinical history or examining a patient, where information from previous tests such as the response to a particular question is available to inform future tests. During this process, the clinician may adjust their threshold for a positive result on the basis of the previous test results. The strength of expectation generated by the previous test results is likely to play a role in how far the clinician adjusts this threshold. Thus, a sequence of four positive responses to directed questions in a history might influence a clinician to lower their threshold for the next question, thereby increasing the sensitivity and decreasing the specificity, compared with if the four previous responses had been negative.

In this study, the test was evaluated in two separate subpopulations in which the main difference was the prevalence of abnormality. This has obvious advantages over two separate studies by controlling for a number of factors that may affect the test performance: the same junior doctors, same radiographers, same reference standard and similar patient-mixes.

Nonetheless, there are two principal limitations relating to the quality of the reference standard (a single radiologist's opinion) and a lack of blinding between the test and the reference standard, raising the potential for review bias. ${ }^{32}$ It is difficult to gauge the effect a lower quality reference standard would have on performance estimates, but it is unlikely to have a differential bias between the high- and low-prevalence populations. Equally, the effects of review bias are likely to inflate estimates of the sensitivity and specificity in both the high- and low-prevalence populations and given it is differences between these performance statistics that are important to demonstrate the principle, inflated estimates in both subpopulations are less of a problem. 
In the regression model, the sensitivity and specificity were treated as independent variables. A bivariate random effects model would maintain the association between the sensitivity and specificity, and individual patient data models have been suggested. ${ }^{33}$ While such advanced approaches may augment the analysis, they would not change the broad findings demonstrated here.

In summary, the diagnostic performance of junior doctors in interpreting $\mathrm{x}$-rays does vary with pre-test probability and this seems to be predominantly based on changing the implicit threshold in response to previous test results. Furthermore, it is unlikely that these findings are confined to the example analysed here. As such, it is an area deserving of further research to establish the extent by which it affects those tests in which there is a subjective element in the execution of the test.

Acknowledgements I would like to thank Dr Shyamaly Sur, MRCOG, in helping collect some of the original data for the study, Professor Chris Hyde, FFPHM, Professor Aneez Esmail, PhD, and Professor Graham Dunn, PhD, for comments on the manuscript. I had full access to all of the data in the study and take responsibility for the integrity of the data and the accuracy of the data analysis.

Contributors BHW conceived the study, interpreted the data and wrote this manuscript.

Funding This research received no specific grant from any funding agency in the public, commercial or not-for-profit sectors. The author was in receipt of a Medical Research Council fellowship during the conduct of this study.

Competing interests The author has completed the Unified Competing Interest form at http://www.icmje.org/coi_disclosure.pdf (available on request from the corresponding author) and declares that (1) BHW had support from a Medical Research Council fellowship during the conduct of this work, (2) has no relationship with any companies that might have an interest in the submitted work in the previous 3 years, (3) spouse, partner or children have no financial relationships that may be relevant to the submitted work and (4) have no non-financial interests that may be relevant to the submitted work

Provenance and peer review Not commissioned; externally peer reviewed.

Data sharing statement No additional data are available.

\section{REFERENCES}

1. Zhou X, Obuchowski NA, McClish DK. Statistical Methods in Diagnostic Medicine. New York: John Wiley and Sons, 2002:21.

2. Feinstein AR. Clinical Epidemiology: The Architecture of Clinical Research. Philadelphia: Saunders, 1985:434-9.

3. Kramer MS. Clinical Epidemiology and Biostatistics: A Primer for Clinical Investigators and Decision-Makers. Berlin: Springer, 1988:211-16.

4. Lalkhen AG, McCluskey A. Clinical tests: sensitivity and specificity, continuing education in anaesthesia. Crit Care Pain 2008;8:221-3.

5. Boyko EJ. Re: "Meta-analysis of Pap test accuracy" (Letter). Am J Epidemiol 1996;143:406-7.

6. Leeflang M, Bossuyt $\mathrm{P}$, Irwig L. Diagnostic test accuracy may vary with prevalence: implications for evidence-based diagnosis. J Clin Epidemiol 2009;62:5-12.

7. Gianrossi R, Detrano R, Colombo A, et al. Cardiac fluoroscopy for the diagnosis of coronary artery disease: a meta analytic review. Am Heart J 1990;120:1179-88.

8. Lachs MS, Nachamkin I, Edelstein $\mathrm{PH}$, et al. Spectrum bias in the evaluation of diagnostic tests: lessons from the rapid dipstick test for urinary tract infection. Ann Intern Med 1992;117:135-40.
9. Lee $\mathrm{KH}$, Hashimoto SA, Hooge JP, et al. Magnetic resonance imaging of the head in the diagnosis of multiple sclerosis: a prospective 2-yea follow-up with comparison of clinical evaluation, evoked potentials, oligoclonal banding, and CT. Neurology 1991;41:657-60.

10. O'Connor PW, Tansey CM, Detsky AS, et al. The effect of spectrum bias on the utility of magnetic resonance imaging and evoked potentials in the diagnosis of suspected multiple sclerosis. Neurology 1996;47:140-4.

11. Dagnelie CF, Bartelink ML, Van Der Graaf $Y$, et al. Towards a better diagnosis of throat infections (with group A beta-haemolytic streptococcus) in general practice. Br J Gen Pract 1998;48: 959-62.

12. DiMattteo LA, Lowenstein SR, Brimhall B, et al. The relationship of pharyngitis and the sensitivity of a rapid antigen test: evidence of spectrum bias. Ann Emerg Med 2001;38:648-52.

13. Hall MC, Kieke B, Gonzales R, et al. Spectrum bias for a rapid antigen detection test for group $A \beta$-haemolytic streptococcus pharyngitis in a paediatric population. Pediatrics 2004; 114:182-6.

14. Wells PS, Anderson DR, Rodger M, et al. Evaluation of D-dimer in the diagnosis of suspected deep-vein thrombosis. $N$ Engl J Med 2003;349:1227-35.

15. Oudega R, Hoes AW, Moons KG. The Wells rule does not adequately rule out deep venous thrombosis in primary care patients. Ann Intern Med 2005;143:100-7.

16. Sultana RV, Zalstein $S$, Cameron $P$, et al. Dipstick urinalysis and the accuracy of the clinical diagnosis of urinary tract infection. J Emerg Med 2001:20:13-19.

17. Jahnke C, Bauer E, Hengge UR, et al. Accuracy of diagnosis of pediculosis capitis: visual inspection vs wet combing. Arch Dermatol 2009;145:309-13.

18. Hudelist $\mathrm{G}$, Oberwinkler $\mathrm{KH}$, Singer $\mathrm{CF}$, et al. Combination of transvaginalsonography and clinical examination for preoperative diagnosis of pelvic endometriosis. Hum Reprod 2009;24:1018-24

19. Taylor KJ, Merritt C, Piccoli C, et al. Ultrasound as a complement to mammography and breast examination to characterise breast masses. Ultrasound Med Biol 2002;28:19-26.

20. Aguilar C, del Villar V. Combined D-dimer and clinical probability are useful for exclusion of recurrent deep venous thrombosis. $A m \mathrm{~J}$ Hematol 2007;82:41-4.

21. Sostman HD, Miniati M, Gottschalk A, et al. Sensitivity and specificity of perfusion scintigraphy combined with chest radiography for acute pulmonary embolism in PIOPED II. J Nucl Med 2008;49:1741-8.

22. Egglin TK, Feinstein AR. Context bias reference: a problem in diagnostic radiology. JAMA 1996;274:1752-5.

23. Willis BH, Sur SD. How good are emergency department senior house officers at interpreting $\mathrm{x}$-rays following radiographers' triage? Eur J Emerg Med 2007;14:6-13.

24. Moses LE, Shapiro D, Littenberg B. Combining independent studies of a diagnostic test into a summary ROC curve: data-analytic approaches and some additional considerations. Stat Med 1993;12:1293-316.

25. Littenberg B, Moses LE. Estimating diagnostic accuracy from multiple conflicting reports: a new meta-analytic method. Med Decis Making 1993;13:313-21.

26. Walter SD. Properties of the summary receiver operating characteristic (SROC) curve for diagnostic test data. Stat Med 2002;21:1237-56

27. Collett D. Modelling Binary Data. 2nd edn. London: Chapman and Hall/CRC, 2003:269-301

28. Gelman A, Hill J. Data Analysis Using Regression and Multilevel/ Hierarchical Models. Cambridge, UK: Cambridge University Press, 2007:301-23.

29. Rogers AW. Textbook of Anatomy. London, UK: Churchill Livingstone, 1992:20-8.

30. Ransohoff DF, Feinstein AR. Problems of spectrum and bias in evaluating the efficacy of diagnostic tests. $N$ Engl J Med 1978;299:926-30

31. Willis BH. Spectrum bias - why clinicians need to be cautious when applying diagnostic test studies. Fam Pract 2008;25:390-6.

32. Whiting $P$, Rutjes AWS, Dinnes J, et al. Development and validation of methods for assessing the quality of diagnostic accuracy studies. Health Technol Assess 2004;8:63.

33. Riley RD, Dodd SR, Craig JV, et al. Meta-analysis of diagnostic test studies using individual patient data and aggregate data. Stat Med 2008;27:6111-36. 